\title{
Exploration of the Regulatory Effects of PSORI- CM01 and Yin Xie Ling on microRNAs in the Peripheral Blood Monocytes of Patients with Blood Stasis Syndrome of Psoriasis Vulgaris
}

Yue Lu

Guangzhou University of Chinese Medicine

Yao Qi

Shanghai Molecular Medicine Engineering Technology Research Center

Li Li

Guangzhou University of Chinese Medicine

Yuhong Yan

Guangzhou University of Chinese Medicine

Jianan Wei

Guangzhou University of Chinese Medicine

Danni Yao

Guangzhou University of Chinese Medicine

Hao Deng

Guangzhou University of Chinese Medicine

Jingwen Deng

Guangzhou University of Chinese Medicine

Shuyan Ye

Guangzhou University of Chinese Medicine

Haiming Chen

Guangzhou University of Chinese Medicine

Qubo Chen

Guangdong Hospital of Traditional Chinese Medicine

Hengjun Gao

Shanghai Molecular Medicine Engineering Technology Research Center

Ling Han ( $\sim$ linghan99@gzucm.edu.cn )

Guangdong Hospital of Traditional Chinese Medicine https://orcid.org/0000-0002-6168-8828

Chuanjian Lu

Guangzhou University of Chinese Medicine 
Research

Keywords: Blood stasis syndrome of psoriasis vulgaris, microRNA expression chip, PSORI-CM01, Yin Xie Ling, mechanism of action

Posted Date: August 26th, 2020

DOI: https://doi.org/10.21203/rs.3.rs-62188/v1

License: (c) (i) This work is licensed under a Creative Commons Attribution 4.0 International License.

Read Full License 


\section{Abstract}

Background: Traditional Chinese medicines (TCMs) have been used to manage psoriasis since thousands of years and are associated with advantages such as long remission period and few side effects. PSORI-CM01 is a clinical TCM formula for treating the blood stasis syndrome of psoriasis vulgaris. It is optimised from Yin Xie Ling, a herbal preparation formulated by the Chinese national medical master Xuan Guowei. In the present study, we used microRNA (miRNA) chip technology to analyse the effects of PSORI-CM01 and Yin Xie Ling on miRNA differential expression in the peripheral blood monocytes of patients with blood stasis syndrome of psoriasis vulgaris.

Methods: Blood samples collected from healthy subjects and patients with blood stasis syndrome of psoriasis vulgaris were obtained from the Department of Dermatology in the Guangdong Provincial Hospital of Chinese Medicine. These samples were obtained before and after treatment with PSORI-CM01 or Yin Xie Ling. Differentially expressed miRNAs were analysed by performing miRNA expression microarray to evaluate the mechanisms underlying the effects of the two formulations.

Results: It was found that hsa-miR-3184-3p, hsa-miR-3653-3p, hsa-miR-20a-3p, hsa-miR-5681b, and hsamiR-26a-5p were differentially expressed between the PSORI-CM01 and healthy control groups as well as between the Yin Xie Ling and healthy control groups before treatment, without any significant differences in their expression after treatment. The results revealed that the therapeutic effect of PSORI-CM01 on psoriasis was mediated mainly via the regulation of the adherens junction, thyroid hormone signalling, and proteoglycans in cancer pathways, whereas that of Yin Xie Ling was mediated mainly via the regulation of the endoplasmic reticulum protein processing, Hippo signalling, adherens junction, thyroid hormone signalling, cell cycle, proteoglycans in cancer, p53 signalling, oocyte meiosis, and unsaturated fatty acid biosynthesis pathways.

Conclusions: The findings demonstrate that PSORI-CM01 and Yin Xie Ling are beneficial for treating blood stasis syndrome of psoriasis vulgaris through the regulation of hsa-miR-20a-3p and hsa-miR-3184$3 p$ expression and, consequently, through the adherens junction, thyroid hormone signalling, and proteoglycans in cancer pathways.

Trial registration: Registration number, ChiCTR-TRC-14005185; Date of registration, 8 August 2014.

\section{Background}

Psoriasis is a chronic, proliferative autoimmune skin disease governed by various genetic factors and stimulated by multiple environmental factors with a global prevalence of $2-3 \%[1,2]$. The characteristic skin lesions in psoriasis are red papules or plaques covered with several layers of accumulated silverwhite scales that can be at a level higher than the skin surface. The boundaries of the rashes are clear, and most cases are accompanied by obvious and unbearable skin itching. Western medicine treatments for psoriasis have several shortcomings, such as short remission periods, easy recurrence and intolerance, and considerable side effects of most drugs [3]. In contrast, traditional Chinese medicine 
(TCM) treatments for the condition are associated with several advantages including long remission periods, stable condition after treatment, rare recurrence, and fewer side effects. Consequently, these TCM treatments have attracted the attention of medical communities in China and abroad in recent years [4]. Abnormal microangiogenesis is closely related to the occurrence, persistence, and recurrence of psoriasis and plays an important role in the pathogenesis of the condition [5].

Psoriasis, also called 'Baibi' in TCM, has been managed with TCM treatments since thousands of years. Syndrome differentiation is the core principle of clinical TCM practice, and it remains the foundation for the modernisation of TCMs [6]. A research group analysed the distribution of syndromes in the literature on psoriasis vulgaris from 1979 to 2010 and found that the top three syndromes-blood heat, blood dryness, and blood stasis-accounted for $75.85 \%$ of all the syndromes [7]. Furthermore, analysis of the correlation between basic TCM syndromes and the stages of and conditions occurring with psoriasis vulgaris revealed the following distribution among patients: blood stasis syndrome $(70.3 \%)>$ blood dryness syndrome $(17.3 \%)>$ blood heat syndrome $(12.4 \%)$. The analysis also clarified that blood stasis syndrome is related to the stable period of the condition [8]. Studies have shown obvious microcirculation disorders, haemorheological changes, and abnormal capillaries characterised by vascular distortion and increased permeability in patients with psoriasis [9-11].

PSORI-CM01 is a clinical TCM formula optimised from Yin Xie Ling, a herbal preparation formulated by the Chinese national medical master and famous TCM master of dermatology Xuan Guowei. It is used to treat blood stasis syndrome of psoriasis vulgaris as it promotes blood circulation, removes blood stasis, cools blood, and promotes detoxification. PSORI-CM01 is composed of Radix Paeoniae Rubra, Chloranthus spicatus, Radices Lithospermi, Curcuma zedoaria, Smilacis glabrae rhizome, smoked plum, and liquorice. In contrast, Yin Xie Ling is composed of Angelica sinensis, Radix Paeoniae Rubra, Chloranthus spicatus, Radix Rehmanniae Recens, Ligusticum wallichii, Radices Lithospermi, Curcuma zedoaria, Smilacis glabrae rhizome, smoked plum, and liquorice. Yin Xie Ling was approved for production by the Guangdong Provincial Hospital of Chinese Medicine (Guangdong, China) in 1998. In 2002, Yin Xie Ling was approved by the Guangdong Provincial Drug Administration as a hospital preparation (Z20080123; patent publication number: CN 105233150A). The clinical efficacy and safety of Yin Xie Ling have been verified in more than 200,000 individuals since more than 20 years [12-14]. After more than 10 years of optimisation of Yin Xie Ling, PSORI-CM01 (patent publication number: CN 101632827A), which has a clear chemical composition and has been assessed using basic experiments and clinical research, was developed [15-17].

The purpose of this study was to clarify the mechanisms underlying the activity of PSORI-CM01 and Yin Xie Ling in the treatment of blood stasis syndrome of psoriasis vulgaris by microRNA (miRNA) expression microarray for the first time. This is important to provide a basis for the clinical development of PSORI$\mathrm{CM} 01$ and to ensure the availability of the treatment to patients.

\section{Materials And Methods}




\section{Clinical experiment}

Blood samples collected from healthy volunteers and patients with blood stasis syndrome of psoriasis vulgaris were obtained from the Department of Dermatology, Guangdong Provincial Hospital of Chinese Medicine, for a randomised, double-blind, double-dummy clinical research [18]. The diagnostic criteria used were in line with those previously reported for psoriasis vulgaris [19]. Additionally, the diagnosis of TCM syndrome type was consistent with the diagnosis of blood stasis syndrome [4]. Table 1 shows detailed information on patients with psoriasis, including demographics and psoriasis area and severity index (PASI).

\section{Drugs}

PSORI-CM01 granules and matching placebo granules were obtained from Tianjiang Pharmaceutical (catalogue no. 1511329; Jiangyin, Jiangsu Province, China). Yin Xie Ling tablets and identical placebo tablets were obtained from Kangyuan Pharmaceutical (catalogue no. 20151003; Guangzhou, Guangdong Province, China). Ultra-high liquid chromatography coupled with electrospray ionisation hybrid linear trap quadrupole Orbitrap mass spectrometry and high-performance liquid chromatography-photodiode array were used to analyse various batches of PSORI-CM01 and Yin Xie Ling, respectively, for quality control purposes, as described previously $[20,21]$.

\section{Group intervention}

One group of participants received $5.5 \mathrm{~g}$ of PSORI-CM01 granules two times daily and five placebo tablets three times daily for 12 weeks (PSORI-CM01 group). Another group received five Yin Xie Ling tablets three times daily and $5.5 \mathrm{~g}$ of placebo granules two times daily for 12 weeks (Yin Xie Ling group). All treatments were administered after meals. Further details on the treatments have been previously reported [18].

\section{Instruments and reagents}

TRIzol reagent (catalogue no. T9424; Sigma, Neustadt, Germany), miRNeasy Micro Kit (catalogue no. T9424 217084; Qiagen, Hilden, Germany), RNase-Free DNase Set (catalogue no. 79254, Qiagen), Tiangen miRcute miRNA cDNA First-Strand Synthesis Kit (catalogue no. KR201; Tiangen, Beijing, China), SYBR Green PCR kit (catalogue no. Fp411-02, Tiangen), Agilent 2100 Bioanalyzer (Agilent Technologies, Santa Clara, CA, USA), Scanner G2505C (Agilent Technologies), and Affymetrix Agilent Human miRNA, Release $21.0(8 \times 60 \mathrm{~K}$; design ID, 070156) chip were used in this study.

\section{Sample collection and processing}


Blood $(10 \mathrm{~mL})$ was collected from the elbow vein of all subjects into anticoagulant tubes containing heparin. Peripheral blood monocytes (PBMCs) were separated using a kit according to the manufacturer's instructions and were then stored at $-80^{\circ} \mathrm{C}$.

\section{RNA extraction and quality control}

Total RNA was extracted using TRIzol reagent according to the manufacturer's protocol and purified using miRNeasy Micro Kit and RNase-Free DNase Set. During each RNA extraction, total RNA sample was used as the positive quality control, whereas RNase-free water was used as the negative quality control. The integrity of purified total RNA was assessed using the Agilent 2100 Bioanalyzer after quality checks were done.

\section{MiRNA chip experiment}

Forty-three PBMCs samples (17 from patients with blood stasis syndrome of psoriasis vulgaris [6 from the PSORI-CM01 group and 11 from the Yin Xie Ling group] before and after treatment each and 9 samples of the healthy control group) were analysed using the Agilent microarray chip (Human miRNA, Release 21.0). Total RNA was determined using the Agilent 2100 Bioanalyzer and quantified using a spectrophotometer (NanoDrop ND-2000; Thermo Scientific, MA, USA). An mRNA quality check was performed, after which mRNA samples were reverse transcribed to cDNA using the Tiangen miRcute miRNA cDNA First-Strand Synthesis Kit. The samples were purified, labelled with cyanine 3-CTP, and placed in the microarray chip for hybridisation, after which the microarray expression was scanned using the Agilent Scanner G2505C.

\section{Data analysis}

Feature Extraction Software (version10.7.1.1, Agilent Technologies) was used to process the microarray scans and extract expression data. GeneSpring software (version 13.1, Agilent Technologies) was then used for quantile standardisation and follow-up processing. The standardised data were filtered, and at least one set of detected labelled probes in each group of samples was subjected to subsequent comparative analysis. Screening of differentially expressed miRNAs was performed using $t$-test $P$ value in the Benjamini-Hochberg procedure with a false discovery rate (FDR-BH) and multiple change value (fold change) after FDR-BH adjustment. The screening criteria were an up- or downregulated multiple change value of at least 1.2 and an FDR-BH value of less than 0.1. Results for the PSORI-CM01 and Yin Xie Ling groups were analysed by paired $t$ test before and after the treatment. In contrast, results for the healthy control group were analysed by two independent-samples $t$-tests. The FDR-BH value was calculated after arranging the $P$ values in ascending order. Unsupervised hierarchical clustering of the differentially expressed miRNAs selected from each group was performed using OmicShareTools. Target mRNAs of the differentially expressed miRNAs were predicted using DIANA Tools and its three databases (TarBasev7.0, microT-CDS [v5.0], and TargetScan). The target mRNAs were then analysed by gene 
ontology and Kyoto Encyclopedia of Genes and Genomes enrichment analyses to determine the biological functions or pathways mainly affected by the differentially expressed miRNAs.

\section{Results}

\section{Differences in miRNAs between the groups}

The results obtained under conditions of FDR-BH values less than 0.1 and |fold change| greater than 1.2 are shown in Table 2. In PBMCs of the PSORI-CM01 and healthy control groups, 17 differentially expressed miRNAs were found before treatment, including 5 miRNAs with upregulated expression and 12 with downregulated expression. Additionally, 16 differentially expressed miRNAs were found after treatment, including 5 miRNAs with upregulated expression and 11 with downregulated expression. However, in PBMCs of the Yin Xie Ling and healthy control groups, 20 differentially expressed miRNAs were found before treatment, including 6 miRNAs with upregulated expression and 14 with downregulated expression, whereas 20 differentially expressed miRNAs were found after treatment, including 4 miRNAs with upregulated expression and 16 with downregulated expression.

\section{Unsupervised hierarchical cluster analysis of differentially expressed miRNAs between the groups}

The results of the unsupervised hierarchical clustering of differentially expressed miRNAs identified in each group are shown in the form of a heat map in Fig. 1. The expression of has-mir6877-3p and has-mir3184-3p in PBMCs was higher in the PSORI-CM01 group than in the healthy control group before treatment (Fig. 1A); however, after the treatment, the expression of only has-mir-6877-3p was higher in the PSORI-CM01 group (Fig. 1B).

Furthermore, the expression of has-mir-3184-3p, has-mir-6877-3p, and has-mir-5681b in PBMCs was higher in the Yin Xie Ling group than in the healthy control group before treatment (Fig. 1C). In contrast, after the treatment, the expression of has-mir-6877-3p, has-mir-3149, and has-mir-5187-5p was higher in the Yin Xie Ling group than in the healthy control group (Fig. 1D).

\section{Wayne analysis of differentially expressed miRNAs}

The Wayne analysis revealed that among the differentially expressed miRNAs between the PSORI-CM01 and healthy control groups, hsa-miR-487a-3p, hsa-miR-4793-3p, hsa-miR-20a-3p, and hsa-miR-3184-3p expression after treatment did not significantly differ from that before treatment. Conversely, there were significant differences in the expression of the following 13 miRNAs, both before and after treatment, between the PSORI-CM01 and healthy control groups: hsa-miR-3130-3p, hsa-miR-3176, hsa-miR-3193, hsa-miR-770-5p, hsa-miR-6776-5p, hsa-miR-6814-5p, hsa-miR-6795-5p, hsa-miR-4750-5p, hsa-miR-4294, hsa-miR-23a-5p, hsa-miR-378f, hsa-miR-374c-5p, and hsa-miR-6877-3p (Fig. 2A). 
The analysis also revealed that among the differentially expressed miRNAs between the Yin Xie Ling and healthy control groups, hsa-miR-3184-3p, hsa-miR-3653-3p, hsa-miR-20a-3p, hsa-miR-5681b, and hsamiR-26a-5p expression after treatment did not significantly differ from that before treatment. In contrast, there were significant differences in the expression of the following 15 miRNAs, both before and after treatment, between the Yin Xie Ling and healthy control groups: hsa-miR-4304, hsa-miR-487a-3p, hsamiR-3130-3p, hsa-miR-3176, hsa-miR-3193, hsa-miR-770-5p, hsa-miR-6776-5p, hsa-miR-6814-5p, hsa-miR6795-5p, hsa-miR-4750-5p, hsa-miR-4294, hsa-miR-23a-5p, hsa-miR-378f, hsa-miR-374c-5p, and hsa-miR6877-3p (Fig. 2B).

Furthermore, hsa-miR-20a-3p and hsa-miR-3184-3p were differentially expressed before treatment and were common between the Yin Xie Ling and PSORI-CM01 groups; however, the expression of these miRNAs in these groups was not significantly different after treatment compared with that in the healthy control group (Fig. 2C). Additionally, the following miRNAs were differentially expressed both before and after treatment in the Yin Xie Ling and PSORI-CM01 groups: hsa-miR-3130-3p, hsa-miR-3176, hsa-miR3193, hsa-miR-770-5p, hsa-miR-6776-5p, hsa-miR-6814-5p, hsa-miR-6795-5p, hsa-miR-4750-5p, hsa-miR4294, hsa-miR-23a-5p, hsa-miR-378f, hsa-miR-374c-5p, and hsa-miR-6877-3p (Fig. 2D).

\subsection{Pathway enrichment analysis for differentially expressed miRNAs}

Figure 3A shows results of the pathway enrichment analysis for hsa-miR-487a-3p, hsa-miR-4793-3p, hsamiR-20a-3p, and hsa-miR-3184-3p, which were differentially expressed between the PSORI-CM01 and healthy control groups before treatment but were not significantly expressed after treatment. Notably, hsa-miR-20a-3p, which participates in the adherens junction, thyroid hormone signalling, and proteoglycans in cancer pathways, was found to be the main miRNA affected by PSORI-CM01.

Figure 3B shows that hsa-miR-3184-3p, hsa-miR-3653-3p, hsa-miR-20a-3p, hsa-miR-5681b, and hsa-miR26a-5p were differentially expressed between the Yin Xie Ling and healthy control groups before treatment; however, their expression after treatment was not significantly different in these groups. Notably, hsa-miR-20a-3p and hsa-miR-26a-5p, which are involved in the endoplasmic reticulum protein processing, Hippo signalling, adherens junction, thyroid hormone signalling, cell cycle, proteoglycans in cancer, p53 signalling, and oocyte meiosis pathways, were the main miRNAs affected by Yin Xie Ling. Additionally, hsa-miR-26a-5p is involved in the unsaturated fatty acid biosynthesis pathway.

\section{Discussion}

Presently, the aetiology and pathogenesis of psoriasis are unknown; however, the consensus among most scholars is that it is a multicellular autoimmune disease [22]. Several genetic and environmental factors are implicated in the pathogenesis of psoriasis. These factors cause imbalances in the expression and secretion of cytokines and chemokines, resulting in cytokine storms, chronic persistent inflammation of the epidermal microenvironment, and consequently, psoriatic dermatitis [23]. Currently, there is no radical 
cure for psoriasis; therefore, TCM preparations are the first-choice treatments for many patients in China [24].

Professor Xuan Guowei highlighted that blood stasis continues throughout the course of psoriasis. He also reported that the treatment of psoriasis should involve the elimination of blood stasis and clearing of collaterals, for which he formulated Yin Xie Ling, a herbal preparation now routinely used to treat psoriasis in the Guangdong Provincial Hospital of Chinese Medicine [12, 25]. After nearly 10 years of research, the preparation has been simplified and optimised to the formula PSORI-CM01 [16, 17]. In our previous study on PSORI-CM01 intervention for patients with blood stasis syndrome of psoriasis vulgaris, PASI score improved by $50 \%$ in $92.7 \%$ of patients after treatment. Moreover, the clinical efficacy of the treatment was satisfactory. Thus, the purpose of the present study was to explore the accurate treatment for psoriasis using TCM preparations and to explain the mechanisms underlying the effects of PSORICM01 and Yin Xie Ling in the treatment of the blood stasis syndrome of psoriasis vulgaris.

MiRNA is a small non-coding RNA composed of $18-25$ nucleotides. It binds to a specific target mRNA and exerts a negative regulatory effect by promoting the degradation of the target mRNA, thereby inhibiting translation. MiRNAs are involved in the regulation of several biological processes such as apoptosis and cell proliferation, differentiation, and development [26, 27]. To date, more than 250 types of miRNAs have been reported to be abnormally expressed in the peripheral blood and skin lesions of patients with psoriasis [28]. Additionally, more than 100 types of abnormally expressed miRNAs have been detected in the serum of patients with plaque psoriasis [29]. Studies have shown that miRNAs participate in the regulation of $\mathrm{T}$ cell differentiation by inhibiting or promoting their differentiation into helper T cells [30]. MiRNA also plays a crucial role in the proliferation and physiological function of regulatory $T$ cells [31]. Consequently, an imbalance in miRNA expression leads to abnormal proliferation of keratinocytes [32].

In the present study, it was shown that the miRNAs that were differentially expressed between the PSORICM01 and healthy control groups before treatment showed no significant difference in their expression after treatment: hsa-miR-487a-3p, hsa-miR-4793-3p, hsa-miR-20a-3p, and hsa-miR-3184-3p. Similarly, hsamiR-3184-3p, hsa-miR-3653-3p, hsa-miR-20a-3p, hsa-miR-5681b, and hsa-miR-26a-5p were differentially expressed between the Yin Xie Ling and healthy control groups before treatment but showed no significant difference in their expression after treatment. Additionally, the differentially expressed miRNAs has-miR-20a-3p and hsa-miR-3184-3p were similar between the PSORI-CM01 and Yin Xie Ling groups. A previous study showed that miR-20a-3p expression is downregulated in psoriatic lesions and interleukin22 -stimulated keratinocytes. The study also indicated that miR-20a-3p can affect the proliferation and apoptosis of keratinocytes by directly targeting SFMBT1 protein and the transforming growth factor$\beta 1 /$ survivin signalling pathway [33]. Another study demonstrated that miR-26a-5p inhibits the proliferation of 3T3-L1 preadipocytes and promotes adipogenic differentiation, which are involved in fat metabolism [34]. 
In the present study, the comparative pathway enrichment analysis for differentially expressed miRNAs and their target mRNAs revealed that the fatty acid biosynthesis, fatty acid metabolism, and steroid biosynthesis pathways were differentially enriched between patients with blood stasis syndrome of psoriasis vulgaris and healthy controls. Additionally, the mechanisms underlying the efficacy of PSORICM01 as a treatment for psoriasis involve regulation of the adherens junction, thyroid hormone signalling, and proteoglycans in cancer pathways, whereas those of Yin Xie Ling involve regulation of the endoplasmic reticulum protein processing, Hippo signalling, adherens junction, thyroid hormone signalling, cell cycle, proteoglycans in cancer, p53 signalling, oocyte meiosis, and unsaturated fatty acid biosynthesis pathways. A retrospective study found that the incidence of metabolic syndrome was higher in patients with psoriasis than in healthy individuals [35]. It has been reported that the incidence of fatty liver is significantly higher in patients with articular psoriasis than in healthy controls [36] and that obesity is significantly correlated with the incidence of psoriasis [37]. In addition, dyslipidaemia has been reported as an important factor in the pathophysiology of psoriasis [38].

There is one limitation to the present study. The detected sample size was small as only 17 patients with blood stasis syndrome of psoriasis vulgaris were included. Therefore, we aim to expand the sample size in our next study. In our future research, we plan to predict treatment effects and formulate personalised TCM treatment plans via analysis of miRNA expression profiles.

\section{Conclusions}

In summary, we compared the expression profiles of miRNAs in the PBMCs of patients with blood stasis syndrome of psoriasis vulgaris, before and after treatment with PSORI-CM01 or Yin Xie Ling, with those of healthy controls. We found that that the fatty acid biosynthesis, fatty acid metabolism, and steroid biosynthesis pathways were differentially enriched between the patients and healthy controls. PSORICM01 mainly affected the adherens junction, thyroid hormone signalling, and proteoglycans in cancer pathways, whereas Yin Xie Ling mainly affected the endoplasmic reticulum protein processing, Hippo signalling, adherens junction, thyroid hormone signalling, cell cycle, proteoglycans in cancer, p53 signalling, oocyte meiosis, and unsaturated fatty acid biosynthesis pathways. Overall, the findings of this

study provide a deeper understanding of the pathogenesis of blood stasis syndrome of psoriasis vulgaris and lay a foundation for elucidating the mechanisms of action of TCMs.

\section{Abbreviations}

TCM, traditional Chinese medicine; miRNA, microRNA; PASI, psoriasis area and severity index; PBMC, peripheral blood monocyte

\section{Declarations}

Acknowledgements 
Not applicable.

\section{Authors' contributions}

All authors have participated extensively in the study and had proofread the final manuscript. CJL, LH and HJG conceived and designed the research. YL and YQ conducted the experiments. YHY, DNY, HD, JWD, and SYY collected patients. QBC helped sample preservation and transportation. YL, YQ, LL, JAW and HMC analyzed the data. YL wrote the manuscript. CJL, LH and HJG approved and reviewed the final manuscript. All authors read and approved the final manuscript.

\section{Funding}

This research was financially supported by the Guangdong Province Science and Technology Planning Project (2017A050506041, 2017B030314166, 2019A1515010636, 2020A1515010607, and 2020B1111100006), Guangdong Provincial Department of Education Project (2018KQNCX046), Guangzhou Science and Technology Project (201807010051), and Guangdong Provincial Hospital of Chinese Medicine Special Fund (YN2018HK01, YN2018ZD08, YN2018RBA02, YN2016XP02, YN2019QJ04).

\section{Availability of data and materials}

The datasets used and/or analyzed during the current study would be available from the corresponding author on reasonable request.

\section{Ethics approval and consent to participate}

The study was performed in accordance with the Declaration of Helsinki and the relevant clinical trial research regulations of China (trial registration number, ChiCTR-TRC-14005185; registration date, 8 August 2014). All the study protocols were in accordance with the ethical standards of Guangdong Provincial Hospital of Chinese Medicine Clinical Research Ethics Committee (ethical approval number, B2012-53-03). Each patient enrolled in the study was required to sign an informed consent form. All participants were informed of the purpose of the study and the treatments before their blood samples were collected.

\section{Consent for publication}

Not applicable.

\section{Competing interests}

The authors declare that they have no competing interests.

\section{References}


1. Perera GK, Di Meglio P, Nestle FO. Psoriasis. Annu Rev Pathol. 2012;7:385-422.

2. Leisner MZ, Riis JL, Schwartz S, Iversen L, Østergaard SD, Olsen MS. Psoriasis and risk of mental disorders in Denmark. JAMA Dermatol. 2019;155(6):745-7.

3. Mourad A, Straube S, Armijo-Olivo S, Gniadecki R. Factors predicting persistence of biologic drugs in psoriasis: A systematic review and meta-analysis. Br J Dermatol. 2019;181(3):450-8.

4. Lu CJ, Yu JJ, Deng JW. Disease-syndrome combination clinical study of psoriasis: Present status, advantages, and prospects. Chin J Integr Med. 2012;18(3):166-71.

5. Hern S, Allen MH, Sousa AR, Harland CC, Barker JN, Levick JR, et al. Immunohistochemical evaluation of psoriatic plaques following selective photothermolysis of the superficial capillaries. $\mathrm{Br}$ J Dermatol. 2001;145(1):45-53.

6. Yan YH, Guo J, Lu CJ. Exploration on TCM research for the treatment of psoriasis based on precision medicine. World J Integr Trad West Med. 2018;33:4789-92.

7. Lu CJ, Zeng Z, Xie XL, Ning J. Distribution of Chinese medical syndrome in ordinary psoriasis: Literature from 1979 to 2010. J Trad Chin Med. 2012;53:959-61 (article in Chinese).

8. Yan YH, Lu CJ, Yao DN, Du H, Li HL. Study on the correlation between the basic syndrome of traditional Chinese medicine and the stage and condition of psoriasis vulgaris. J Guangzhou Univ Trad Chin Med. 2012;4:22-6 (article in Chinese).

9. Creamer D, Allen MH, Sousa A, Poston R, Barker JN. Localization of endothelial proliferation and microvascular expansion in active plaque psoriasis. Br J Dermatol. 1997;136(6):859-65.

10. Bhushan M, Mclaughlin B, Weiss JB, Griffiths CE. Levels of endothelial cell stimulating angiogenesis factor and vascular endothelial growth factor are elevated in psoriasis. $\mathrm{Br} \mathrm{J}$ Dermatol. 1999;141(6):1054-60.

11. Rosenberger C, Solovan C, Rosenberger AD, Jinping L, Treudler R, Frei U, et al. Upregulation of hypoxia-inducible factors in normal and psoriatic skin. J Invest Dermatol. 2007;127(10):2445-52.

12. Liao LH, Huang YJ, Fan RQ, Xuan GW. Observation on therapeutic effect of 47 cases of psoriasis vulgaris (stagnation skin type) treated by promoting blood circulation. New J Trad Chin Med. 2005;37:40-1.

13. Wang $\mathrm{L}$, Huang YJ, Wang MH. Clinical observation of Yinxiling tablet in treating psoriasis vulgaris. J Guangzhou Univ Trad Chin Med. 2009;26:520-5 (article in Chinese).

14. Deng JQ, He W, Wei JA, Liu QZ, Wu HM. Intervention effects of Yinxieling tablets on leptin expression of obese patients with psoriasis vulgaris. World Chin Med. 2019;14:166-9.

15. Lu CJ, Xiang Y, Xie XL, Xuan ML, He ZH. A randomized controlled single-blind clinical trial on 84 outpatients with psoriasis vulgaris by auricular therapy combined with optimized Yinxieling Formula. Chin J Integr Med. 2012;18(3):186-91.

16. Wei JA, Han L, Lu CJ, Zhao RZ, Sun J, Lu Y, et al. Formula PSORI-CM01 eliminates psoriasis by inhibiting the expression of keratinocyte cyclin B2. BMC Complement Altern Med. 2016;16:255. 
17. Han L, Sun J, Lu CJ, Zhao RZ, Lu Y, Lin HJ, et al. Formula PSORI-CM01 inhibits the inflammatory cytokine and chemokine release in keratinocytes via NF-kB expression. Int Immunopharmacol. 2017;44:226-33.

18. Deng J, Yao D, Lu C, Wen Z, Yan Y, He Z, et al. Oral Chinese herbal medicine for psoriasis vulgaris: Protocol for a randomised, double-blind, double-dummy, multicentre clinical trial. BMJ Open. 2017;7(11):e014475.

19. Boehncke WH, Schön MP. Psoriasis. Lancet. 2015;386(9997):983-94.

20. Chen YJ, Zhao RZ, Zhao Y, Lu CJ. Simultaneous determination of chlorogenic acid, peoniflorin, isofraxidin, ferulaic acid and astilbin in Yinxieling liquid extract by HPLC-PDA. Chin Trad Pat Med. 2010;32:1522-5.

21. Chen SD, Lu CJ, Zhao RZ. Identification and quantitative characterization of PSORI-CM01, a Chinese medicine formula for psoriasis therapy, by liquid chromatography coupled with an LTQ Orbitrap mass spectrometer. Molecules. 2015;20(1):1594-609.

22. Menter A, Gottlieb A, Feldman SR, Van Voorhees AS, Leonardi CL, Gordon KB, et al. Guidelines of care for the management of psoriasis and psoriatic arthritis: Section 1. Overview of psoriasis and guidelines of care for the treatment of psoriasis with biologics. J Am Acad Dermatol. 2008;58(5):826-50.

23. Kaushik SB, Lebwohl MG. Psoriasis: Which therapy for which patient: Psoriasis comorbidities and preferred systemic agents. J Am Acad Dermatol. 2019;80(1):27-40.

24. Yan YH, Lu CJ. Optimized Yinxieling for treatment of psoriasis vulgaris: An exploratory clinical trial. Trad Chin Drug Res Clin Pharmacol. 2011;22:691-3.

25. Zhong JB, Yin X, Lu CJ, Xuan GW. Introduction of Professor Xuan Guowei's experience in treating psoriasis. New J Trad Chin Med. 2004;9:11-2.

26. Yekta S, Shih IH, Bartel DP. MicroRNA-directed cleavage of HOXB8 mRNA. Science. 2004;304(5670):594-6.

27. Borchert GM, Lanier W, Davidson BL. RNA polymerase III transcribes human microRNAs. Nat Struct Mol Biol. 2006;13(12):1097-101.

28. Hawkes JE, Nguyen GH, Fujita M, Florell SR, Callis Duffin K, Krueger GG, et al. MicroRNAs in psoriasis. J Invest Dermatol. 2016;136(2):365-71.

29. Pivarcsi A, Meisgen F, Xu N, Ståhle M, Sonkoly E. Changes in the level of serum microRNAs in patients with psoriasis after antitumour necrosis factor-a therapy. Br J Dermatol. 2013;169(3):56370.

30. Talebi F, Ghorbani S, Chan WF, Boghozian R, Masoumi F, Ghasemi S, et al. MicroRNA-142 regulates inflammation and $\mathrm{T}$ cell differentiation in an animal model of multiple sclerosis. J Neuroinflamm. 2017;14(1):55.

31. Zhang L, Ke F, Liu Z, Bai J, Liu JL, Yan S, et al. MicroRNA-31 negatively regulates peripherally derived regulatory T-cell generation by repressing retinoic acid-inducible protein 3. Nat Commun. 2015;6:7639. 
32. Suwanwongse K, Shabarek N. miRNA125b Downregulation: a review of the novel paradigm of psoriasis epigenetic regulation. Cureus. 2020;12(1):e6798.

33. Li R, Qiao M, Zhao X, Yan J, Wang X, Sun Q. MiR-20a-3p regulates TGF- $\beta 1 /$ survivin pathway to affect keratinocytes proliferation and apoptosis by targeting SFMBT1 in vitro. Cell Signal. 2018;49:95-104.

34. Geng HR, He H, Tan XT, Guo DG, Ma YJ, Zhang Y, et al. Effects of miR-26a-5p on proliferation and differentiation of 3T3-L1 preadipocyte. Chin J Vet Sci. 2018;38:1962-81.

35. Augustin M, Reich K, Glaeske G, Schaefer I, Radtke M. Co-morbidity and age-related prevalence of psoriasis: Analysis of health insurance data in Germany. Acta Derm Venereol. 2010;90(2):147-51.

36. Russolillo A, lervolino S, Peluso R, Lupoli R, Di Minno A, Pappone N, et al. Obesity and psoriatic arthritis: From pathogenesis to clinical outcome and management. Rheumatol Oxf Engl. 2013;52(1):62-7.

37. Engin B, Kutlubay Z, Yardımcı G, Vehid HE, Ambarcıoğlu P, Serdaroğlu S, et al. Evaluation of body composition parameters in patients with psoriasis. Int J Dermatol. 2014;53(12):1468-73.

38. Pietrzak A, Michalak-Stoma A, Chodorowska G, Szepietowski JC. Lipid disturbances in psoriasis: An update. Mediators Inflamm. 2010;2010:535612.

\section{Tables}

Table 1 Clinical serum samples for miRNA analysis

\begin{tabular}{cccccc}
\hline & $\begin{array}{c}\text { Healthy controls } \\
(\mathbf{N}=\mathbf{9})\end{array}$ & $\begin{array}{c}\text { PSORI-CM01 group } \\
\text { 0 week (N=6) }\end{array}$ & $\begin{array}{c}\text { PSORI-CM01 group } \\
\text { 12 weeks (N=6) }\end{array}$ & $\begin{array}{c}\text { Yin Xie Ling group } \\
\text { 0 week (N=11) }\end{array}$ & $\begin{array}{c}\text { Yin Xie Ling group } \\
\text { 12 weeks (N=11) }\end{array}$ \\
\hline Age & $39.52 \pm 10.31$ & $42.37 \pm 11.28$ & $42.37 \pm 11.28$ & $43.58 \pm 12.53$ & $43.58 \pm 12.53$ \\
\hline Sex N (\%) & & & & & 6 \\
Male & 5 & 3 & 3 & 6 & 5 \\
Female & 4 & 3 & 3 & 5 & $6.53 \pm 4.27$ \\
\hline PASI & $/$ & $7.21 \pm 3.16$ & $6.37 \pm 3.86$ & $7.13 \pm 2.58$ & 6 \\
\hline
\end{tabular}

Table 2 Differentially expressed miRNAs between the groups 
Sample size

\begin{tabular}{|c|c|c|c|c|}
\hline & & miRNA & miRNA & miRNA \\
\hline $\begin{array}{l}\text { PSORI-CM01 group } 0 \text { week vs } \\
\text { healthy control group }\end{array}$ & 6 vs 9 & 17 & 5 & 12 \\
\hline $\begin{array}{l}\text { PSORI-CM01 group } 12 \text { weeks vs } \\
\text { healthy control group }\end{array}$ & 6 vs 9 & 16 & 5 & 11 \\
\hline $\begin{array}{l}\text { Yin Xie Ling group } 0 \text { week vs } \\
\text { healthy control group }\end{array}$ & 11 vs 9 & 20 & 6 & 14 \\
\hline $\begin{array}{l}\text { Yin Xie Ling group } 12 \text { weeks vs } \\
\text { healthy control group }\end{array}$ & 11 vs 9 & 20 & 4 & 16 \\
\hline
\end{tabular}

\section{Figures}



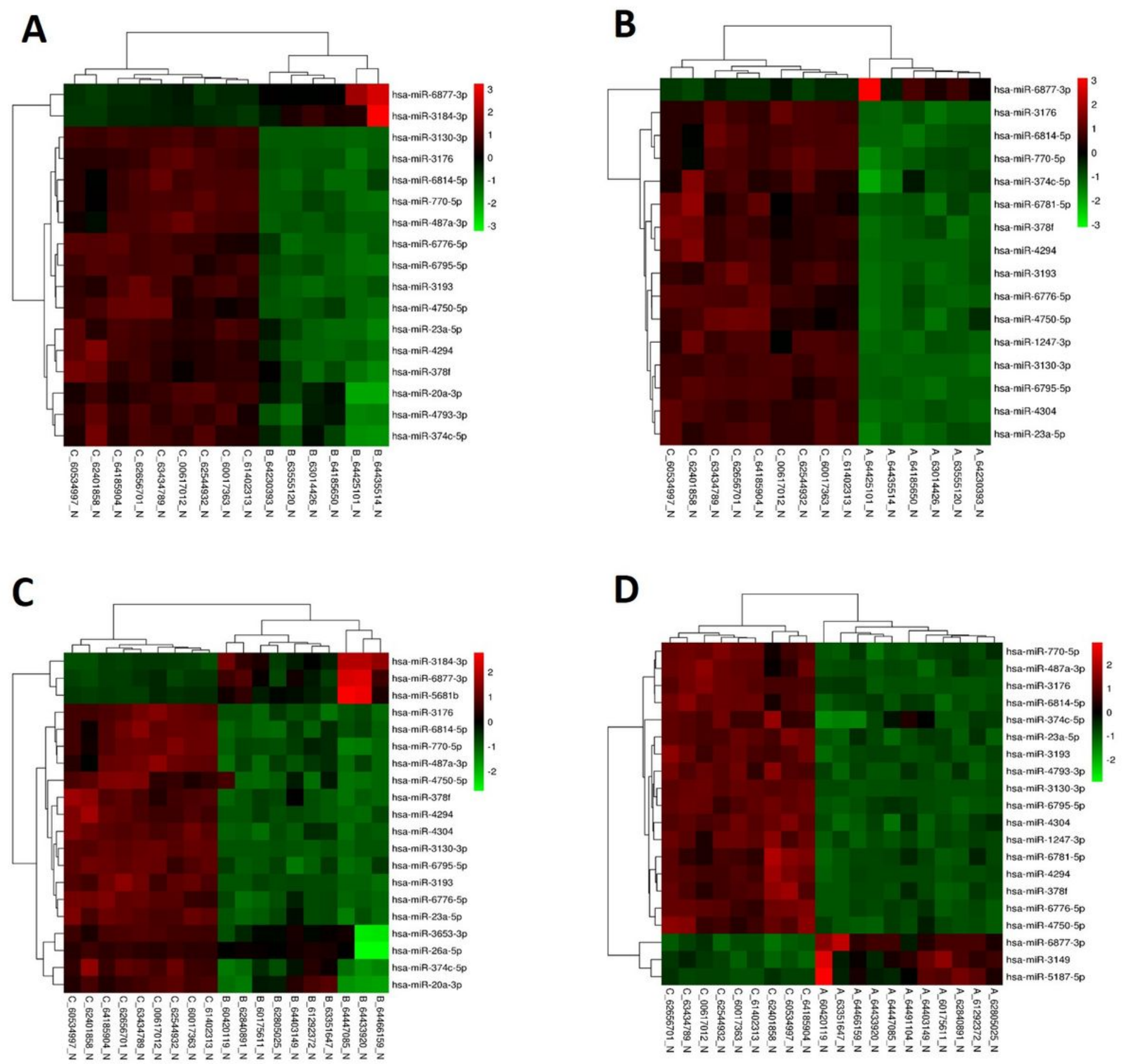

\section{Figure 1}

Unsupervised hierarchical cluster analysis of differentially expressed miRNAs between groups. (a) PSORICM01 group before treatment versus healthy control group. (b) PSORI-CM01 group after treatment versus healthy control group. (c) Yin Xie Ling group before treatment versus healthy control group. (d) Yin Xie Ling group after treatment versus healthy control group. 
A

PSORI-CM01 group 0 week

vs

healthy control group

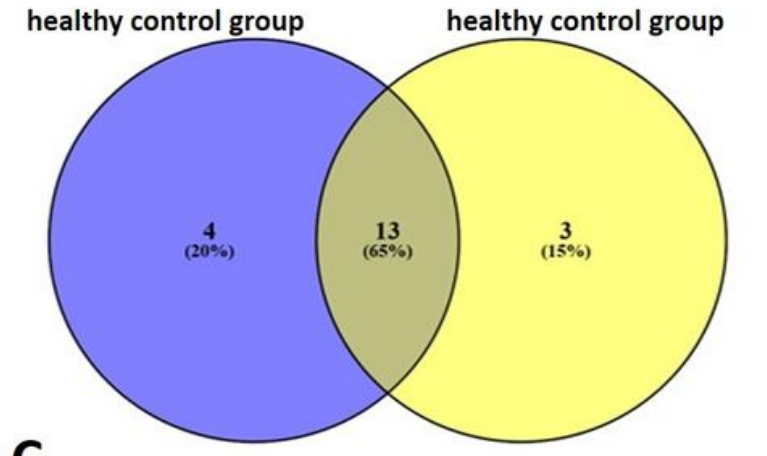

C

PSORI-CM01 group

vs healthy control group

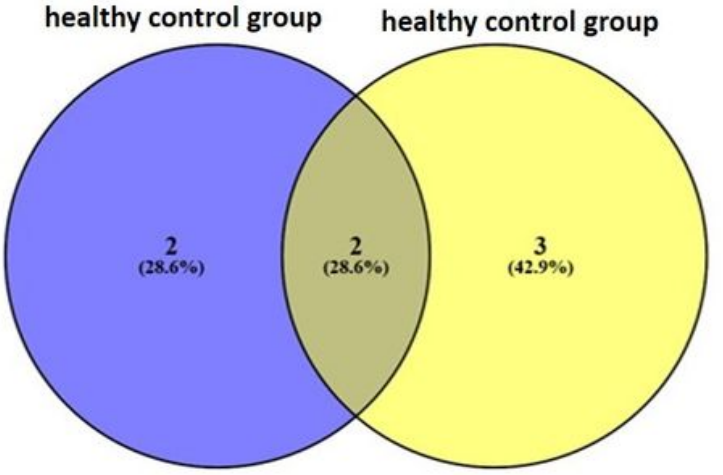

B

Yin Xie Ling group 0 week Yin Xie Ling group 12 weeks

vs vs

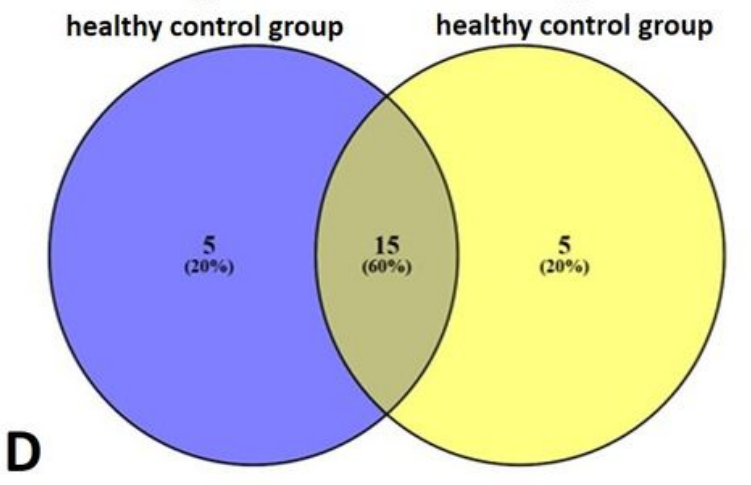

PSORI-CM01 group

vs

Yin Xie Ling group

vs

healthy control group healthy control group

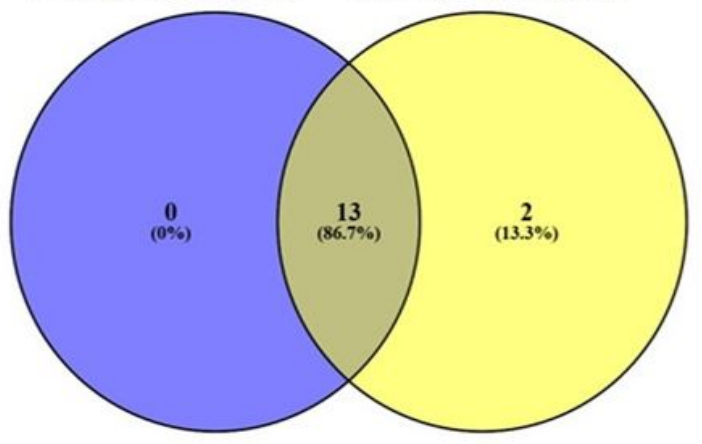

Figure 2

Wayne analysis of differentially expressed miRNAs between the groups. (a) Differential expression of miRNAs between the PSORI-CM01 and healthy control groups before and after treatment. (b)

Differentially expressed miRNAs between the Yin Xie Ling and healthy control groups before and after treatment. (c) Differentially expressed miRNAs before treatment that showed no significant difference in their expression after treatment when compared with their respective expression in the healthy control group. These miRNAs were similar between the PSORI-CM01 and Yin Xie Ling groups. (d) miRNAs that were differentially expressed both before and after treatment compared with that in the healthy control group; these miRNAs were similar between the PSORI-CM01 and Yin Xie Ling groups. 


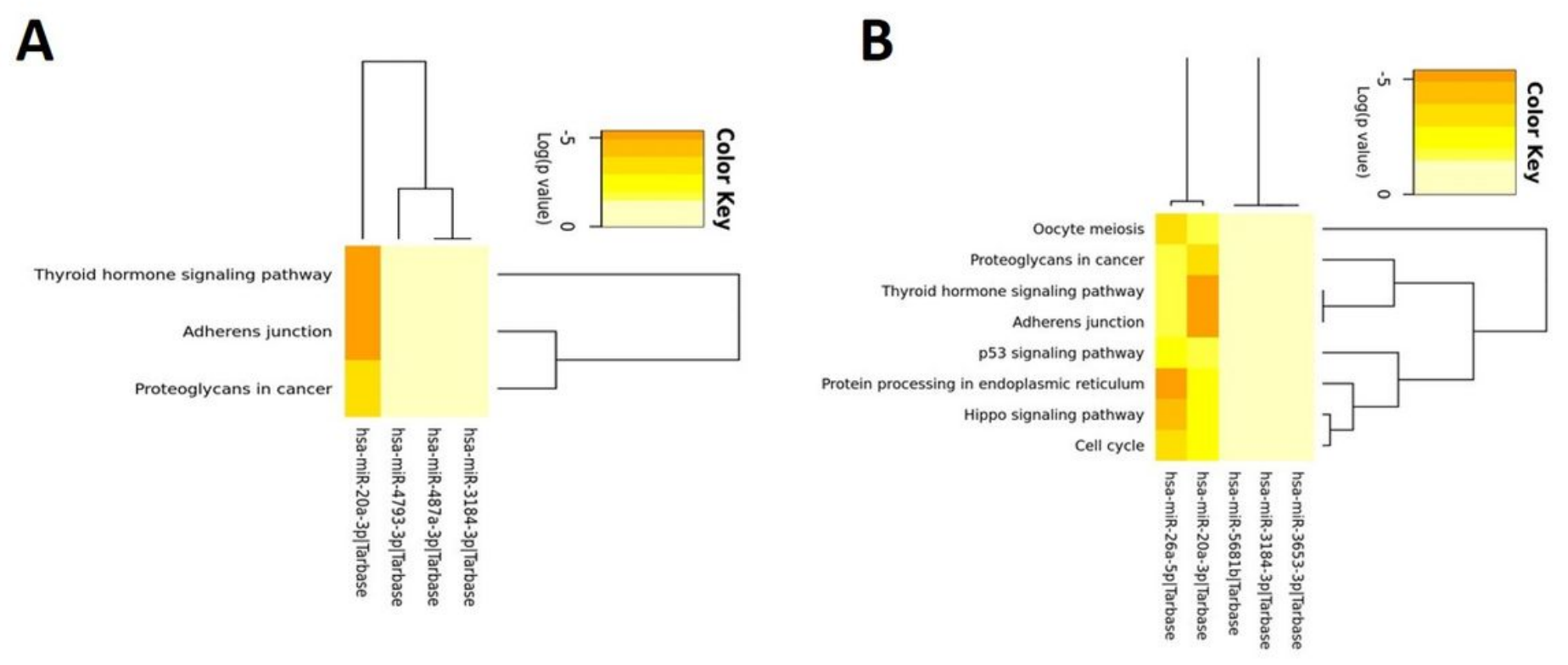

Figure 3

Pathway enrichment analysis of differentially expressed miRNAs between groups. (a) miRNAs that were differentially expressed between the PSORI-CM01 and healthy control groups before treatment exhibited no significant difference in their expression after treatment. (b) miRNAs that were differentially expressed between the Yin Xie Ling and healthy control groups before treatment exhibited no significant difference in their expression after treatment. 